\title{
The virtual history of tourism: from the remnants of life in Penghu Hua-Zai village
}

\author{
Y.-C. Lin ${ }^{1}$, T.-C. Wu ${ }^{2}$, M.-F. Hsu ${ }^{3}$ \& L.-Y. Huang ${ }^{3}$ \\ ${ }^{I}$ College of Planning and Design, \\ National Cheng Kung University, Taiwan \\ ${ }^{2}$ Department of Construction Engineering, \\ National Quemoy University, Taiwan \\ ${ }^{3}$ Department of Architecture, National Cheng Kung University, Taiwan
}

\begin{abstract}
Hua-Zai is a village on Penghu Wangan Island, located on the east side of the Taiwan Strait of Taiwan. West of the village near the coast, north, east and south surround on three sides to hold a gentle hill. That is the traditional village-type of Han Chinese immigrants facing the sea settlement. Locals call the quiet Hua-Zai Village, by its name Jung-She, or "Flower Village", this name first appeared in the Qing Dynasty in 1699, learned on the basis of local genealogy, the late Ming having recorded that ancestors settled. It is believed to have existed as a settlement since Han Chinese from mainland China began settling there three centuries ago. The Hua-Zai Village obtained the valuable World Monuments Watch (WMF) announcing that it is on the "List of 100 most endangered sites", and recognized that the settlement has been there for three centuries of the Chinese Han people immigration and cultural development, in 2004. The new designation for Hua-Zai Village may finally bring about much-needed government support for the challenging work of preserving the houses in the village, which is considered the largest and most well-preserved early Han settlement in Taiwan. The islands geographical environment restricts and decides the life appearance. Hua-Zai Village's geographical environment limits agriculture cultivation, but the fishery resources are rich, the settlement keeping the Han traditional houses, temples, cowshed, water wells, the wharves and the industrial structures. Those architectural styles are regarded as the model of the Han people's traditional settlement, which dates back 75 to 150 years, have not been modified and reflect designs handed down from early Han settlers. Village
\end{abstract}


maintenance is traditional, the farming and fishing way, facing up to bad living conditions, the performance fusion on the diverse geographical environment and the building structure. Lifestyle is keeping a relic of ancient times. In recent years, as more than half of the village's approximately 151 old houses were damaged, although some 50 structures still remain intact, the landscape has partly been marred by the damaged buildings. Even today, some of the remaining 100 villagers - mostly senior citizens and children - live the same way their ancestors did, fishing, collecting seaweed and growing their own food. Despite the settlement's long history, the houses' unique architectural features and historical significance were not appreciated. There still maintains the old wisdom of living in harmony with nature that is valuable, and deserves to be preserved, protected and passed on. The tourists can not understand the meaning of settlement construction and lifestyle. Hence, this study wants to use the humanities histories display on the geographical environment, and humanities history's evolution and geographical environment's vicissitude. In order to reach this plan target, reference to human geography research and old photographs, measured every house, recorded details of their decoration and analyzed the skillful way the coral stones were stacked and held together by a lime and mud mortar. Moreover, by using 3D laser scanner Collected spatial data for drawing 3D models and edit the script, which judged the buildings original appearance and simulated lifestyle. Construct Virtual History of Tourism - to return from the remnants of the Hua-Zai Village life.

Keywords: Penghu Jung-She village, settlement life history, virtual tour, spatial information, modelling.

\section{Introduction}

\subsection{Research background}

"Hua-Zai" is the old name for Zhongshe Village, Wangan Township, Penghu County. It is located on the shore on the south side of Tiantai Mountain. Hua-Zai is a traditional settlement formed by Han immigrants, and the earliest name arose under Emperor Kangxi of the Qing Dynasty in 1699. However, according to the local genealogy, during the reign of Tianqi near the end of the Ming Dynasty, there were settlers, meaning that there is a history of over three hundred years here. Hua-Zai District is located at the center of Wangan Island in Penghu, with a small hill at the center of the settlement, which the locals refer to as the stamen of the flower. The stamen is surrounded by five hills, like a flower. The local elderly state that if one looks from a distance in the sea, the hills look like flower petals surrounding the heart of the flower. Thus, the name of Hua-Zai settlement came about because it looks like a flower from the distance. In 1946, also because Hua-Zai is located at the center of Wangan Island, it was renamed Zhongshe Village. However, a walk in the Zhongshe settlement still allows one to find door plaques from the Hua-Zai era.

The Hua-Zai settlement is in a cluster village form, the core area has the shore to the west, while the north, east, and south are surrounded by slow-rising hills, 
making it a village cluster settlement that faces the ocean. The residents live in a space facing the ocean with hills at their backs, which is a basic arrangement common to traditional settlements in Penghu, revealing the close relationship between people and natural environments. Penghu Hua-Zai is like an island settlement, in which there are insufficiencies in terms of climate, earth, and water supply. The unique landscape and appearance of island culture have created the structural content of the settlement. Over three hundred years of immigrant lifestyles are evinced in geography, geomancy, traditional beliefs, extensive agriculture, seafaring, customs and habits, industrial content, and architecture, forming a cultural life with ocean characteristics [1, 2] (figs. 1 and 2).

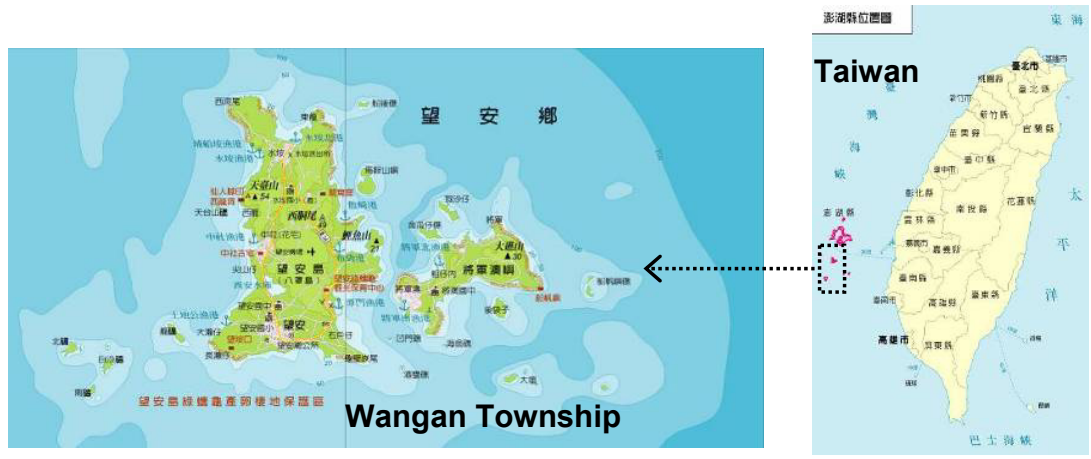

Figure 1: $\quad$ Location of Hua-Zai in Wangwan township and Zhongshe village.
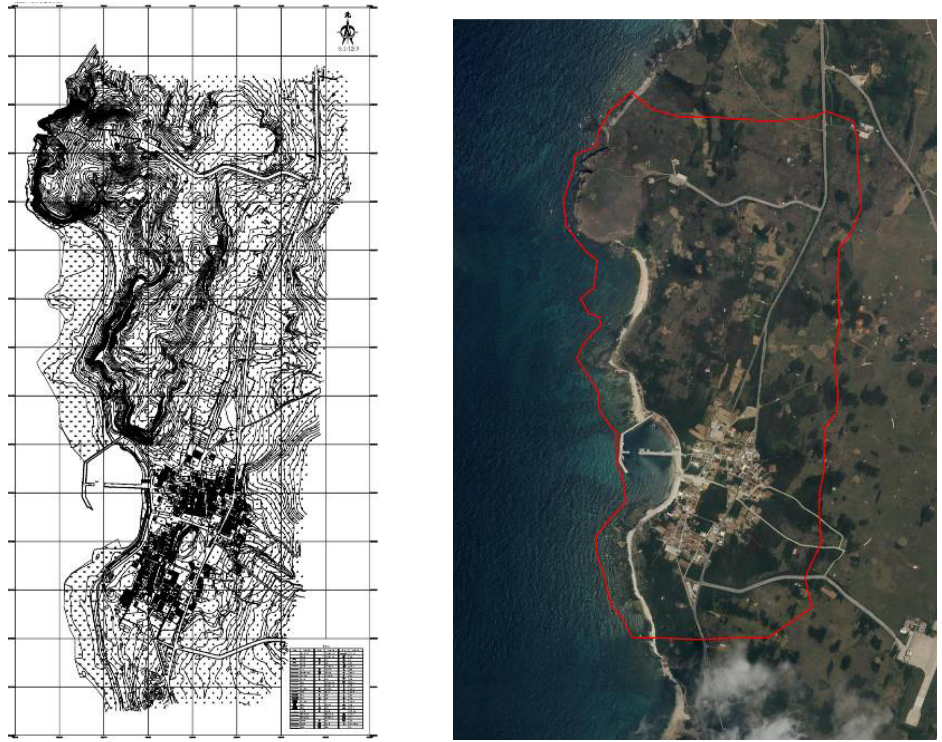

Figure 2: $\quad$ Location of Zhongshe village in Wangwan township (2010). 


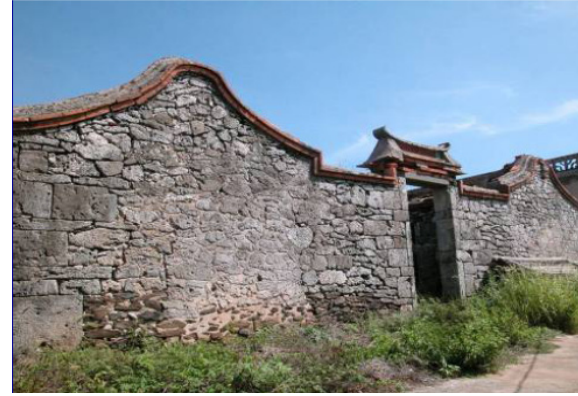

Figure 3: Hua-Zai houses use coral stones as the main material for the wall.

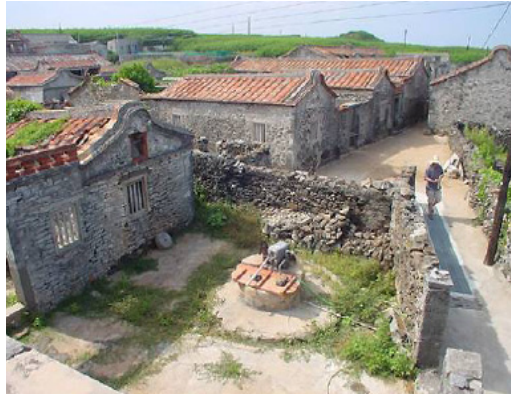

Figure 4: Courtyard house, Wai Tsai Nei, a well of HuaZai settlement.

The geographic environment of the island conditioned and determined lifestyles. The Hua-Zai settlement maintained traditional ways of living such as extensive agriculture and seafaring. The content of life accentuated in the face of an adverse living environment is expressed and blended in diverse geographic environment and architectural buildings. For instance, structures for agricultural production include honeycomb fields, wells, embankment, fertilizer pit, oil cars; structures for fishery production include fish traps and fish cooking sites; structures for animal husbandry include livestock pens; structures for industrial production include cement kilns and brick kilns (figs. 3 and 4). Furthermore, there are settlement spatial structures such as traditional houses, temples, plazas, churches, piers, and popsicle factories, arranged with organic paths among them. Hua-Zai settlement structures have continued and accumulated, even developing local secondary and tertiary industries such as coralline limestone, peanut residue, seafood processing; the life wisdom in using limited resources to continue life fully reflects the environmental characteristics and life philosophy of Hua-Zai in Penghu [3].

After the Second World War, many residents moved to Magong or Taiwan, and only about 100 people currently live in the settlement village of Hua-Zai. According to the pilot study in 2008, there are 139 traditional winged-courtyard style houses in the Hua-Zai settlement, 2 Japanese style houses, and 19 modern houses, for a total of 160; only 52 are complete $[4,5]$. Until now, despite the three hundred year history of the settlement, traditional houses are mostly dilapidated or have collapsed due to disuse (fig. 5). Fortunately, most houses have not been reconstructed and thereby maintained the traditional settlement appearance. The villagers also maintained some of the traditional ways of life, with the early lifestyles of local residents jointly constructed by tangible cultural assets and intangible cultural assets $[6,7]$. 

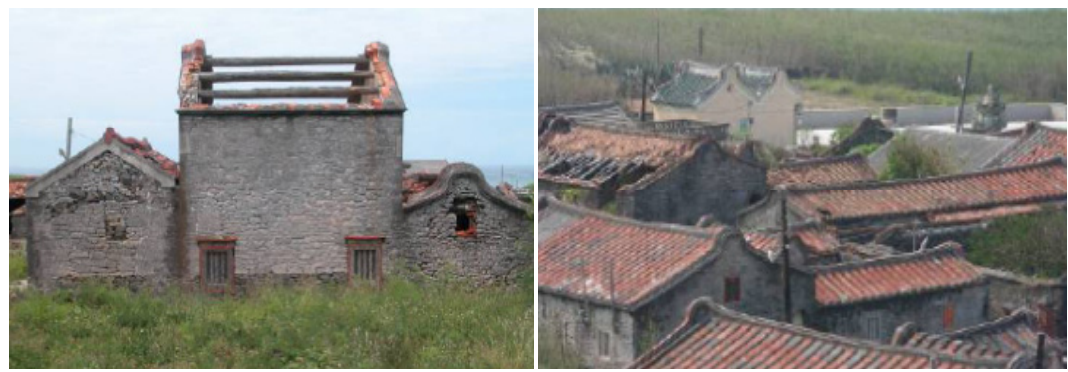

Figure 5: $\quad$ The traditional houses are collapsed as they remain vacant (2008).

\subsection{Research purpose}

Hua-Zai's cultural asset value has been ascertained by "World Monuments Watch (WMF)", in 2004 it placed Hua-Zai on the list of 100 most endangered sites, certifying that the Hua-Zai settlement is the result of three centuries of Han Chinese immigration and cultural development. The geographical environment limited agricultural pursuits, but there are abundant fishery resources. The settlement contains traditional Han Chinese houses, temples, cow pens, wells, piers, and industrial structures. The Hua-Zai settlement is seen as a typical Han settlement due to its existing completeness and diverse architectural styles.

The hundred villagers who currently live in Hua-Zai have mostly maintained the lifestyles of their ancestors, such as fishing, collecting seaweed, and planting for extensive agriculture. This is a microcosm of the features of intangible cultural asset living, overlaying tangible cultural asset houses and settlement environment; the Hua-Zai settlement has value jointly constructed from tangible cultural assets and intangible cultural assets [8-11] (fig. 6).

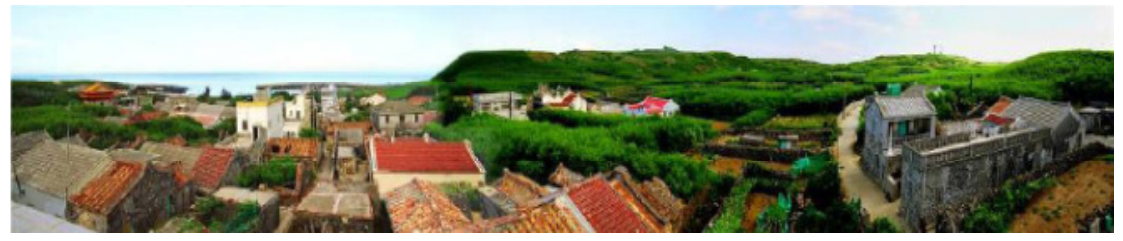

Figure 6: FigHua-Zai Settlement and Mt. Hutou in the north (2008).

Thus, since Hua-Zai has historical human and cultural settlement relics, the purpose of this paper is to archive the traditional settlement life history of Penghua Hua-Zai. The temporal axis is the early days of Japanese occupation in 1895 as representing late Qing relics. The life space, production methods, and cultural features of Hua-Zai residents are used to capture important snippets of human and cultural life on the island, as well as the regional stories created by local life; a four-dimensional method is used to reconstruct a virtual historical tour in Hua-Zai (fig. 7). 


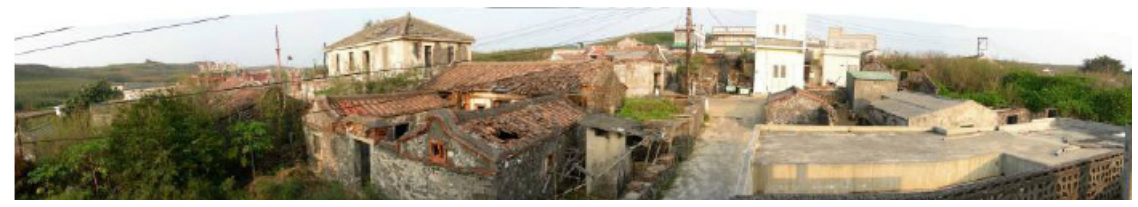

Figure 7: The architecture, structure, and plaza layout belong to organic type paths (2008).

\section{Research objectives and methods}

There are three main types of data being established in this study. First, the large-scale spatial data and minute representative land features are digitized to establish 3D digital spatial data on landform and land feature; then, with the spatial data, numerical landform model data, and 3D model are overlaid, and the geographical information system is used as the basis for the data platform, establishing Hua-Zai 3D landscape platform, providing functions of storage, editing, searching, and exhibition; third, the presentation of virtual reality animation requires literature collection, survey, and on-site interviews to create a theme script, with roles and scenes to be designed, which are then incorporated into the digital accomplishments to construct the 3D model, editing the animation and then outputting the display.

\subsection{D digital spatial data on landform and land feature}

Other than the macro view of the landform model, minute land features are also important spatial data. Representative landscape and land feature in Hua-Zai are selected, including streets, architecture, wells, honeycomb fields, fishing ports, piers, and natural landscape in the Hua-Zai settlement. FARO Photon 80 3D laser scanner is used to obtain color point cloud data, to establish spatial data with spatial coordinates (fig. 8). As for the instances where cover resulted in incomplete point cloud models, the tool plug-in provided by Point Cloud is used on Auto CAD, to directly import 3D point cloud data into Auto CAD (fig. 9). The precise drawing in Auto CAD is used, in reference to the $3 \mathrm{D}$ cloud point location to directly draw the parts that were incompletely scanned, to strengthen the incomplete scans due to on-site cover, and the function of pasting simulated images is used to strengthen visual simulation. 


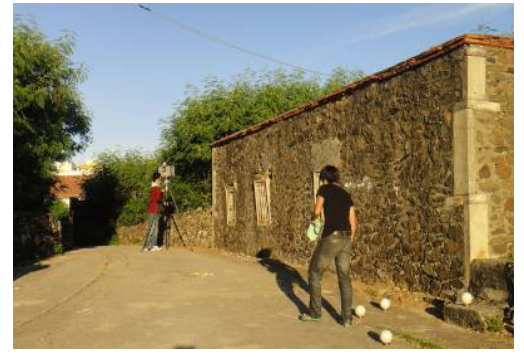

Figure 8: $3 \mathrm{D}$ laser scanner is used to obtain point cloud data.

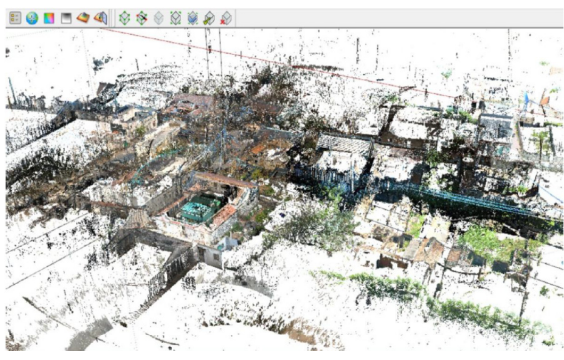

Figure 9: $\quad$ Import the 3D point cloud into $3 \mathrm{D}$ modeling software.

\subsection{D landscape platform}

Collection of spatial data is divided into landform and landscape. According to the "2004 Map Catalogue" printed by Material Production Center, Armaments Bureau, Ministry of National Defense, Wangan Island has two landform maps, 9219-I and 9319-II 1/5000. The landform portion is based on the latest digital aerial colored photography, and Global Positioning System (GPS) is used to collect on-site 3D landform coordinates, to construct Wangan Island digital terrain model (DTM); integration of the two allows for construction of a largescale 3D color landform model for Wangan Island. This model reflects the simulated 3D conditions of Wangan Island.

Original files of colored numerical maps taken by the Aerial Survey Office of Wangan Island on April 15, 2009 are used. 5 meter DEM is used to conduct orthophoto processing, the orthophoto resolving power is $25 \mathrm{~cm}$, producing a numerical landform model (as in the following image), then the Zhongshe HuaZai settlement $1 / 200$ ratio scale numerical landform model is encoded to a 1 meter numerical landform model to produce a 3D color landform model.

Since the data archived in the database is the basic content, one of the research purposes of this plan is to demonstrate added value. Data storage, editing, searching, and exhibition are the basic functions of the system platform for this plan. 3D presentation is used to reproduce the 3D humanities landscape platform for Hua-Zai at the end of the Qing Dynasty. The overall point and technique of archiving is to show a large amount of spatial space, matched with attribute data and supported with audiovisual data (figs. 10 and 11). 


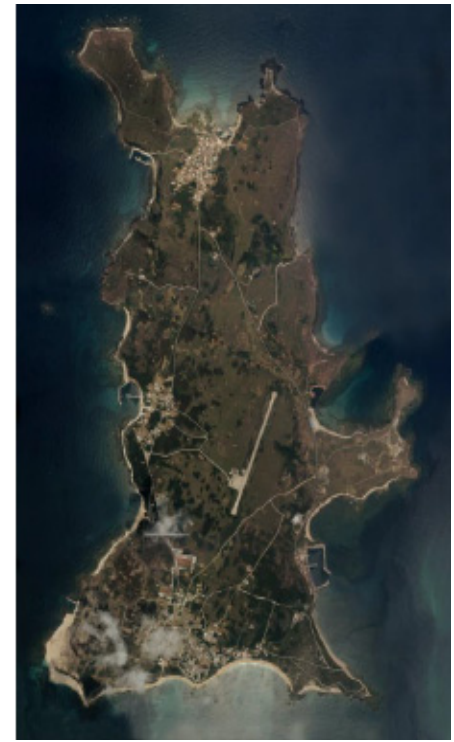

Figure 10: Ortho-image of Penghu.

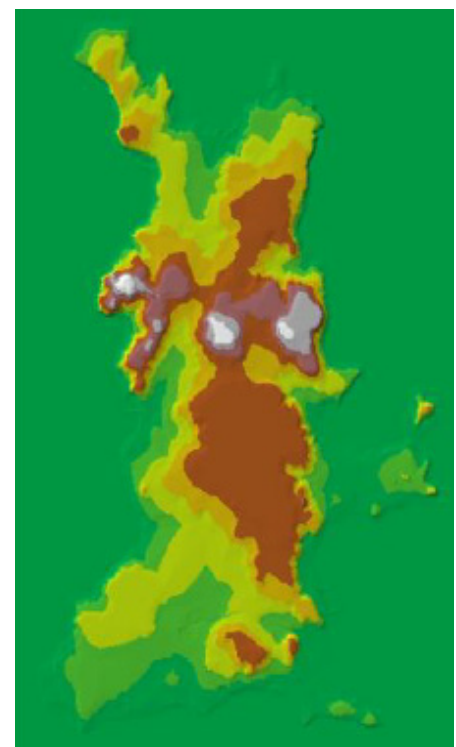

Figure 11: Numerical topographical model of Penghu.

\subsection{Construct 3D model}

Since Hua-Zai landform and land features mix the historical trajectory of the original appearance, expansion, and reconstruction. Without old images as reference, on-site photography and 3D laser scanning are used to obtain the current materials and sizes of landform and land features are obtained (figs. 12 and 13). Reference and evidence-checking of historical materials are used to

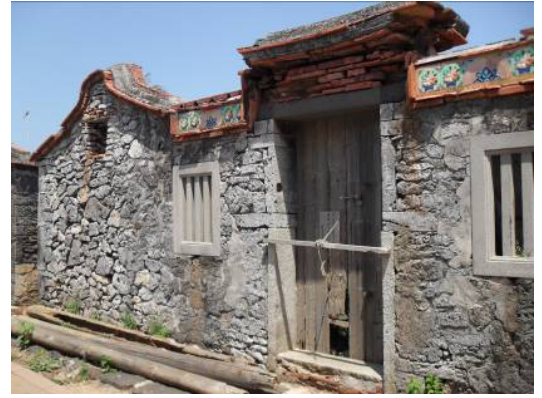

Figure 12: Shooting architecture style and space for model making.

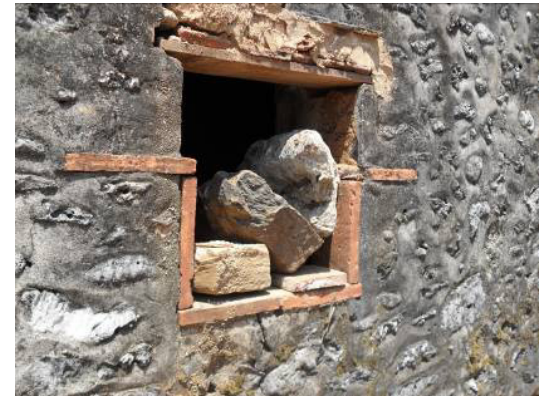

Figure 13: The details of the photos are used for subsequent collage. 
construct a 3D model of the original appearance. This study uses Pointools plugin for SktchUp developed by the English company Pointools, a point cloud modeling software plugin, which directs imports point cloud data into SktchUp, to construct point cloud into a 3D model (fig. 14).
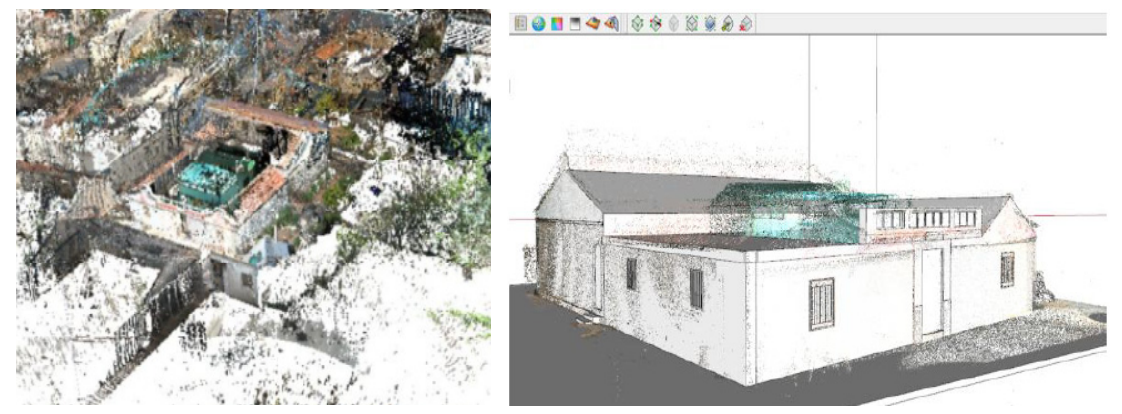

Figure 14: Import 3D point cloud into the 3D modeling software. Use the coordinate of point cloud to draw the $3 \mathrm{D}$ model directly on $3 \mathrm{D}$ point cloud.

The flow for 3D model construction is as follows: 1) on-site photography and recording of formal details, photography is not only taken of the architectural forms and the spatial environments to construct a model, and at the same time the details are photographed for use in follow-up material pasting; 2) 3D laser scan of buildings is used to establish 3D point cloud image data, importing the point cloud data into $3 \mathrm{D}$ modeling software. Point cloud $3 \mathrm{D}$ coordinate data is used to directly draw 3D models in point cloud; 3) after the on-site photograph materials are modified again, the high-resolution images are pasted, or the material paste pieces of point cloud are cut to be laid over the $3 \mathrm{D}$ model, achieving a more realistic result (fig. 15).

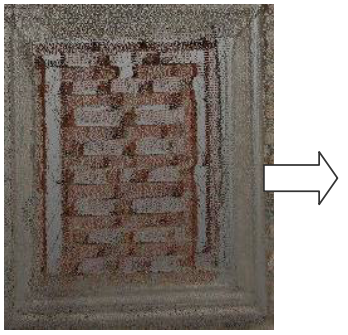

step.1

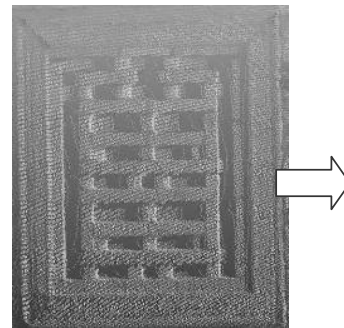

step. 2

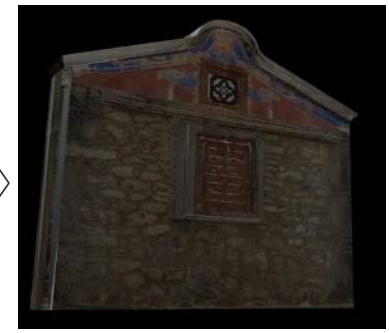

step. 3

Figure 15: Imports point cloud data into SktchUp to construct a 3D model. 


\section{Reappearance of digital historical trajectory in Wangan Hua-Zai}

This work focuses on the integration of attribute data and spatial data, emphasizing the interactive relationship between regional distribution features, humanities phenomena, and geographical environment, therefore it is necessary to construct a script framework for humanities, historical, and environmental change, guiding the modification and simulation of basic data in the spatial database.

\subsection{Concept of digital archive}

Generally speaking, human cultural environment includes customs, ways, culture, and timeline are focused on humanities and history, and is an attribute database. Humanities research recording methods are like traditional anthropological or settlement study investigation methods, via text, pictures, images, and sounds, which can all be placed in GIS attribute database, and would very much conform to the database format and format. Geographical environment includes geology, hydrology, landform, houses, and map classifications; in the past these are displayed on paper or as electronic maps, but now the technology has allowed for representation of $3 \mathrm{D}$ spatial information, archived as 3D data (fig. 16).
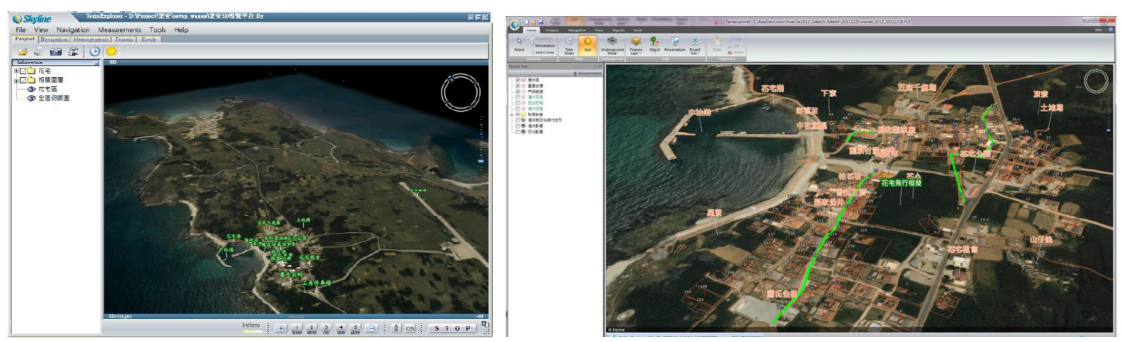

Figure 16: Presentation of the 3D landscape platform with storage, editing, inquiring, and display.

The digitized objects in physical archives include the public spaces in the central area of the settlement (streets and temples), landscape elements of the central areas of the settlement (trees, honeycomb fields, wells, civilian facilities), religious buildings, industrial buildings, residential buildings, images, and documents. The output list of digital archive objects include, 300 photographs of architecture in the Hua-Zai settlement, 5 photographs of the basic image of the Hua-Zai settlement, 3D landscape model images and photos of the center of the settlement, 3D models of 361 buildings in the Hua-Zai settlement, and the landform constructed scenes of the 3D landscape platform. This digital data supports script editing for production of the $3 \mathrm{D}$ animation, to construct historical stories on life, industry, architectural construction, and religious festivals in the 
settlement, to simulate and display the historical trajectory and life in humanities and culture of Hua-Zai.

\subsection{Virtual tourism exhibit}

The reappearance of the historical trajectory of Hua-Zai is also a part of the addition of value through digitization. Basic materials for producing a script include 3D point cloud, 3D model, old photographs, and historical image postproduction $3 \mathrm{D}$ guide animation. In addition, it is necessary to collect existing literature and reports, investigate the humanities and historical data in order to create the script and 3D model. The scenes for Hua-Zai are edited with Lumion, then is imported into SketchUpto create a 3D model, and Revit directly exports the rendered animation. Finally, voice over and subtitles are used as explanations to supplement the figures and activities, combined with geographical data, then the animation is edited to show the virtual travel context (fig. 17).
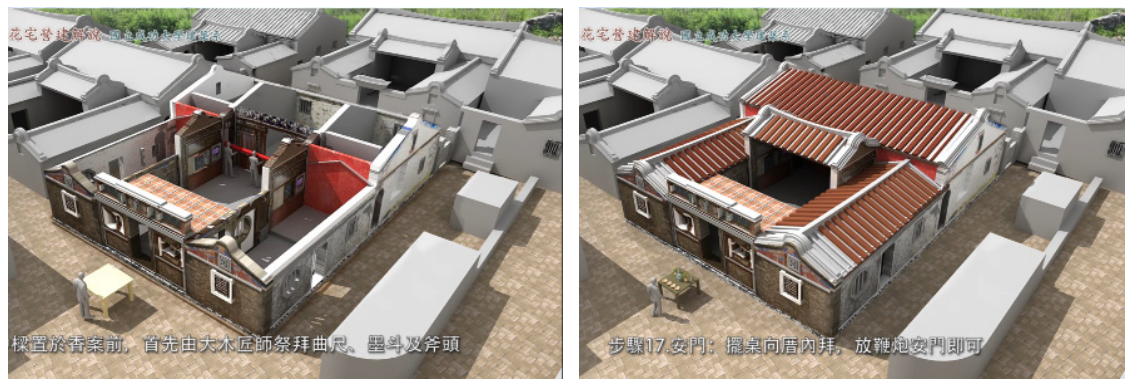

Figure 17: Virtual tourism exhibit of Hua-Zai traditional winged-courtyard style house construction process.

\section{Conclusion}

This paper attempts to use 3D methods to allow living sites that have disappeared to reappear. The guiding model of travel in virtual contexts are used to introduce Penghu Hua-Zai ocean living and culture, so that travelers can use this guide platform to better understand local culture and humanistic value, to provide another kind of travel experience.

The presentation of virtual travel must undergo stringent evidentiary investigation of materials in humanities and history to edit a script with a story to honestly and comprehensively present the feelings in virtual travel. In addition, the meaning behind virtual travel is the concept of digital archive, which not only codes and archives existing born digital media, but also archives old photographs, texts, books, and relics by turning them into digitized objects, to ensure the future usability, durability, and intelligent integration of digital data.

Virtual historical tourism was originally conceived to provide a place to experience the past temporal and spatial context. However, results of VR (virtual 
reality) created by virtual travel can also use AR (augmented reality) digitization technology to project objects or scenes that do not actually exist on designated real space. This allows the traveller to experience virtual images that pass through time and space in real travel sites.

\section{Acknowledgement}

The paper would like to thank "National Science Council/Taiwan Digital Archives Expansion Project (Project number: NSC 99-2631-H-006-005- )" for the provision of research funding.

\section{References}

[1] Chen, C. H., Penghu Islands, Fuming Agricultural Geographical Research Institute: Taipei City, pp.3-11, 1955.

[2] Tseng, L. T., Penghu Islands (Ocean Paradise of Taiwan), Penghu Fengguag Editorial Commission: Kaohsiung, pp.48-50, 1987.

[3] Lin, W. C., Wu, H. F., Traditional Industrial Agriculture of Penghu, Cultural Affairs Bureau, Penghu County: Penghu, 2003.

[4] Taipei National University of the Arts, Project for Checking Historic Buildings in Penghu County (No. 2: Magong City, Wangan Township, Qimei Township), Cultural Affairs Bureau, Penghu County: Penghu, 2002.

[5] Foundation of Historic City Conservation and Regeneration, Pilot Project for Hua-Zai Settlement Preservation Foundation Survey and Community Building, Foundation of Historic City Conservation and Regeneration: Tainan, 2006.

[6] Hsu, J. M. ed., Walking through the Past - Album of Old Photographs in Penghu, Penghu County Cultural Center: Penghu, pp.159-177, 1995.

[7] Lin, G. B. ed., Appearances of the White Sands, Penghu County Cultural Center: Penghu, 1997.

[8] Chang, Y. J., Treasures of Boat Owners - Ocean Oral History of the Penghu Elderly, Cultural Affairs Bureau, Penghu County: Penghu, pp.8-15, 2000.

[9] Chi, L. M. ed., Treasures of Old Photographs in Penghu - Life History Images: Album of Old Photographs, Cultural Affairs Bureau, Penghu County: Penghu, 29-179, 2006.

[10] Chang, Y. J., Penghu Fishing Village, Agriculture and Fisheries, Penghu County Government: Penghu, pp.36-42, 78-81, 2007.

[11] Chi, L. M. ed., Compilation of Old Photographs from Wangan Hua-Zai Settlement, Cultural Affairs Bureau, Penghu County: Penghu, 2009. 